\title{
Publicly funded overactive bladder drug treatment patterns in Ontario over 15 years: An ecologic study
}

Mina Tadrous ${ }^{1}$; Dean Elterman²; Wayne Khuu ${ }^{3}$; Muhammad M. Mamdani ${ }^{4}$; David N. Juurlink³; Tara Gomes ${ }^{4}$

${ }^{1}$ Ontario Drug Policy Research Network (ODPRN) and Leslie Dan Faculty of Pharmacy-University of Toronto; ${ }^{2}$ Division of Urology, Department of Surgery, University Health Network, University of Toronto; ${ }^{3}$ Institute for Clinical Evaluative Sciences; ${ }^{4}$ St. Michael's Hospital; Toronto, ON, Canada

Cite as: Can Urol Assoc J 2017 Dec. 22; Epub ahead of print. http://dx.doi.org/10.5489/cuaj.4541

Published online December 22, 2017

$* * *$

\section{Abstract}

Introduction: Medication is an important option for patients with overactive bladder (OAB), with four different drugs approved over the last 10 years including the first non-anticholinergic treatment, mirabegron. We set out to describe the number and rate of users of medication for the management of $\mathrm{OAB}$ over the last 15 years among residents of Ontario, Canada covered by the public drug programs.

Methods: We conducted a population-based, repeated cross-sectional study examining quarterly publically-funded prescription claims for OAB medications from January 2000 to June 2016 in Ontario, Canada.

Results: We report two major changes in prescription patterns for OAB. The first was the rise of newer, more selective anticholinergics (tolterodine, solifenacin, and darifenacin) replacing oxybutynin. This led to a $54.8 \%$ reduction in the rate of users of oxybutynin over the study period from 10.4 users/1,000 beneficiaries in 2000 to 4.7 users/1,000 beneficiaries in 2016 . Recently we saw the emergence of mirabegron as the most commonly prescribed treatment for OAB. By the final quarter of the observation period mirabegron was the most commonly used OAB treatment with $25.0 \%(n=19,411)$ of all OAB medication users in Ontario $(n=77,660)$. Conclusion: Our findings highlight the rapid uptake of novel agents and a major shift in the treatment of $\mathrm{OAB}$ over the last 3 years. 


\section{Introduction}

Overactive bladder (OAB) is a common condition that is associated with urgency and frequency of urination, urge incontinence, and nocturia occurring mostly in older individuals. ${ }^{1-3}$ OAB has been shown to have a negative effect on physical functioning and quality of life. With the aging population and increased life-expectancy there has been an increase in OAB diagnosis., ${ }^{4,5}$ Medications are second-line treatments for OAB, after non-pharmacological options, with disease management generally relying on the use of anticholinergic agents. ${ }^{6}$ Currently in Canada there are six anticholinergic drugs approved for treatment of OAB symptoms: darifenacin, fesoterodine, oxybutynin, solifenacin, tolterodine and trospium. Three (darifenacin, fesoterodine and mirabegron) of these were approved in the last 10 years. Newer anticholinergics differ from older agents in formulations, dosing schedules and side-effect profiles (i.e. dry mouth, dizziness, and constipation). ${ }^{6,7}$ Anticholinergics were the only medications indicated to treat OAB until the approval of mirabegron in 2013 and its listing on the public drug formulary in 2015. Mirabegron is a beta-3 adrenoceptor agonist and is the first alternative to anticholinergics for the treatment of OAB. The Ontario Public Drug Programs (OPDP) has allowed liberal access to all OAB treatments differing from many other provincial and private payers which limit access. With the changing dynamic of treatment options and the continued growth in the users of OAB medications, we set out to describe the treatment patterns for OAB in Ontario over the last 15 years.

\section{Methods}

We conducted a population-based, repeated cross-sectional study examining quarterly prescription claims for OAB treatments reimbursed by the Ontario Public Drug Programs (OPDP) from January 1, 2000 to June 30, 2016. Drug coverage through the OPDP is available to all residents 65 years of age and older, those with financial needs (due to high drug costs and/or low income) and those living in long-term care homes or requiring homecare. Prescription claims for patients who receive medications through private coverage or pay out of pocket are not captured. In Ontario, over $75 \%$ of OAB drug prescriptions were paid by the public drug program. $^{7}$

All publically-funded drug claims were identified using the Ontario Drug Benefit (ODB) database, which contains records for all prescriptions dispensed to eligible Ontario residents. This dataset was linked using encoded identifiers and analyzed at the Institute for Clinical Evaluative Sciences (ICES). The number and rate of users per drug was reported and plotted to identify trends over time. The rate of users was adjusted per 1,000 eligible ODB beneficiaries per quarter. We also reported the relative market share by total users of each treatment annually. The study was approved by the Institutional Review Board at Sunnybrook Health Sciences Centre, Toronto, Canada.

\section{Results}

Over the 15-year study period there were 10,131,681 prescription claims for publically funded $\mathrm{OAB}$ treatments dispensed in Ontario. The number of users per year more than tripled from 
24,148 users in the first quarter of 2000 to 77,660 users in the second quarter of 2016. There was a steady rate of growth over this study period with an average annual rate of growth of $7.3 \%$ (Figure 1).

Over the study period there has been a large shift in the relative market share of each treatment (Figures 1 and 2). In the first quarter of 2000, the vast majority of users were treated with oxybutynin (68.6\%; 10.4 users/1,000 beneficiaries) with the only other treatments being flavoxate (17.7\%; 2.7 users/1,000 beneficiaries) and tolterodine (13.7\%; 2.1

users/1,000 beneficiaries). Tolterodine use increased 13.7\% from Q1-2000 (2.1 users/1,000 beneficiaries) to Q3-2011(15.3 users/1,000 beneficiaries) before the introduction of solifenacin (2011), darifenacin (2011), and mirabegron (2015) led to a steady reduction in tolterodine use. Between Q4-2011 and Q2-2016, the rate of tolterodine use fell 56.1\%, from 15.5 users/1,000 beneficiaries to 6.8 users/1,000 beneficiaries. In Q2-2015, following its introduction to the public drug formulary, there was a rapid uptake of mirabegron. By the final quarter of the observation period (Q2-2016), mirabegron was the most commonly used OAB treatment (25.0\%) in Ontario with 7.6 users/1,000 beneficiaries (25.0\% of all OAB medication users).

\section{Discussion}

In this population based study of trends in OAB medication treatment over the past 15 years in Ontario, we found that the rate of use of these products has increased considerably more than three-fold. Furthermore, three major changes in prescribing have occurred; the first change occurred in 2004 with the rise of tolterodine which quickly replaced oxybutynin as the most commonly used treatment, the second change was the introduction of newer anticholinergics (solifenacin, darifenacin) which decreased tolterodine use, and the third change was the introduction of mirabegron which led to a rapid uptake of this drug. Mirabegron is now the most commonly used OAB treatment in Ontario.

The observed shifts in $\mathrm{OAB}$ treatment patterns are likely influenced by two factors. First, newer agents are generally perceived to be superior to existing treatments in terms of dosing regimens and side-effect profiles. ${ }^{8}$ The introduction of tolterodine allowed for fewer daily doses (once or twice daily) compared to oxybutynin (three to four-times daily). This improvement was thought to lead to improved adherence and decreased pill burden. ${ }^{9}$ Secondly, newer anticholinergics introduced (tolterodine, solifenacin and darifenacin) were likely perceived to be more tolerable than oxybutynin due to their increased specificity for muscarinic receptors. ${ }^{10}$ This increased specificity is thought to reduce the rate and severity of adverse events and improve tolerability. ${ }^{10}$ These perceived improvements were highly marketed by manufacturers as major reasons to use newer agents over oxybutynin, especially among older patients. With a median age of 73 among Ontario OAB users ${ }^{7}$ a large number of users were shifted away from older treatments by prescribers. This reasoning has also been used to support the early adoption of mirabegron which, because of its different mechanism of action, has a different side effect profile lacking the typical anticholinergics effects such as dry mouth and constipation. Since the Ontario listing allows access to all treatments the results of this study show a strong prescribing preference for newer agents. 
Currently, Ontario has one of the most liberal listing for OAB treatments with all treatments funded by the OPDP. Oxybutynin is listed as General Benefit and all other agents (darifenacin, fesoterodine, solifenacin, tolterodine, trospium and mirabegron), only require a limited use code on prescriptions. In contrast most public payers across Canada and internationally restrict or do not fund newer more costly treatments. The results of our study support the rapid shifting of use to newer agents when access is granted, which in turn will increase spending. These results are important to payers as they plan any listing changes to this drug class.

Our study is not without limitations and the results of this analysis must be interpreted in the context of its study design. Firstly, our study does not include claims paid for by private insurers or out-of-pocket by patients. However, the majority of OAB patients are over the age of 65 and all Ontario residents above the age of 65 received drug coverage. Past research by our team found that over 75\% of OAB drug prescriptions in Ontario in 2014 were paid by the public drug program. ${ }^{7}$ Secondly, we cannot rule out the possibility that some of these treatments may be used off-label for other non-OAB indications. For example, there may be a small proportion of users receiving oxybutynin for hyperhidrosis. However, off-label uses are not common and are not anticipated to greatly impact our findings.

\section{Conclusion}

Treatment patterns for OAB in Ontario have shifted over the past 15 years, with the emergence of mirabegron - a novel agent in this class - as the most commonly prescribed treatment. Our findings highlight the market expansion for these treatment classes and the potential for rapid uptake of novel agents with new mechanisms of action particularly when existing medications have considerable side effects. 


\section{References}

1. Abrams P, Cardozo L, Fall M, et al. The standardisation of terminology in lower urinary tract function: report from the standardisation sub-committee of the International Continence Society. Urology. 2003;61(1):37-49.

2. Harris SS, Link CL, Tennstedt SL, Kusek JW, McKinlay JB. Care seeking and treatment for urinary incontinence in a diverse population. J Urol. 2007;177(2):680-684.

3. Marinkovic S, Rovner E, Moldwin R, al e. The management of overactive bladder syndrome. BMJ. 2012;344:e2365.

4. Buckley BS, Lapitan MC. Prevalence of urinary and faecal incontinence and nocturnal enuresis and attitudes to treatment and help-seeking amongst a community-based representative sample of adults in the United Kingdom. Int J Clin Pract. 2009;63(4):568573.

5. Gorina Y, Schappert S, Bercovitz A, Elgaddal N, Kramarow E. Prevalence of incontinence among older americans. Vital Health Stat 3. 2014(36):1-33.

6. Bettez M, Le Mai Tu KC, Corcos J, Gajewski J, Jolivet M, Bailly G. 2012 update: Guidelines for adult urinary incontinence collaborative consensus document for the Canadian Urological Association. Canadian Urological Association Journal. 2012;6(5):354.

7. Treatments for Overactive Bladder - Pharmacoepidemiology Report. Ontario Drug Policy Research Network;2016.

8. Chapple CR, Cardozo L, Nitti VW, Siddiqui E, Michel MC. Mirabegron in overactive bladder: a review of efficacy, safety, and tolerability. Neurourology and urodynamics. 2014;33(1):17-30.

9. Veenboer PW, Bosch JR. Long-term adherence to antimuscarinic therapy in everyday practice: a systematic review. The Journal of urology. 2014;191(4):1003-1008.

10. Maman K, Aballea S, Nazir J, et al. Comparative efficacy and safety of medical treatments for the management of overactive bladder: a systematic literature review and mixed treatment comparison. European urology. 2014;65(4):755-765. 


\section{Figures and Tables}

Fig. 1. Rate of publically funded overactive bladder treatment users per 1000 eligible in Ontario from 2000-2016.

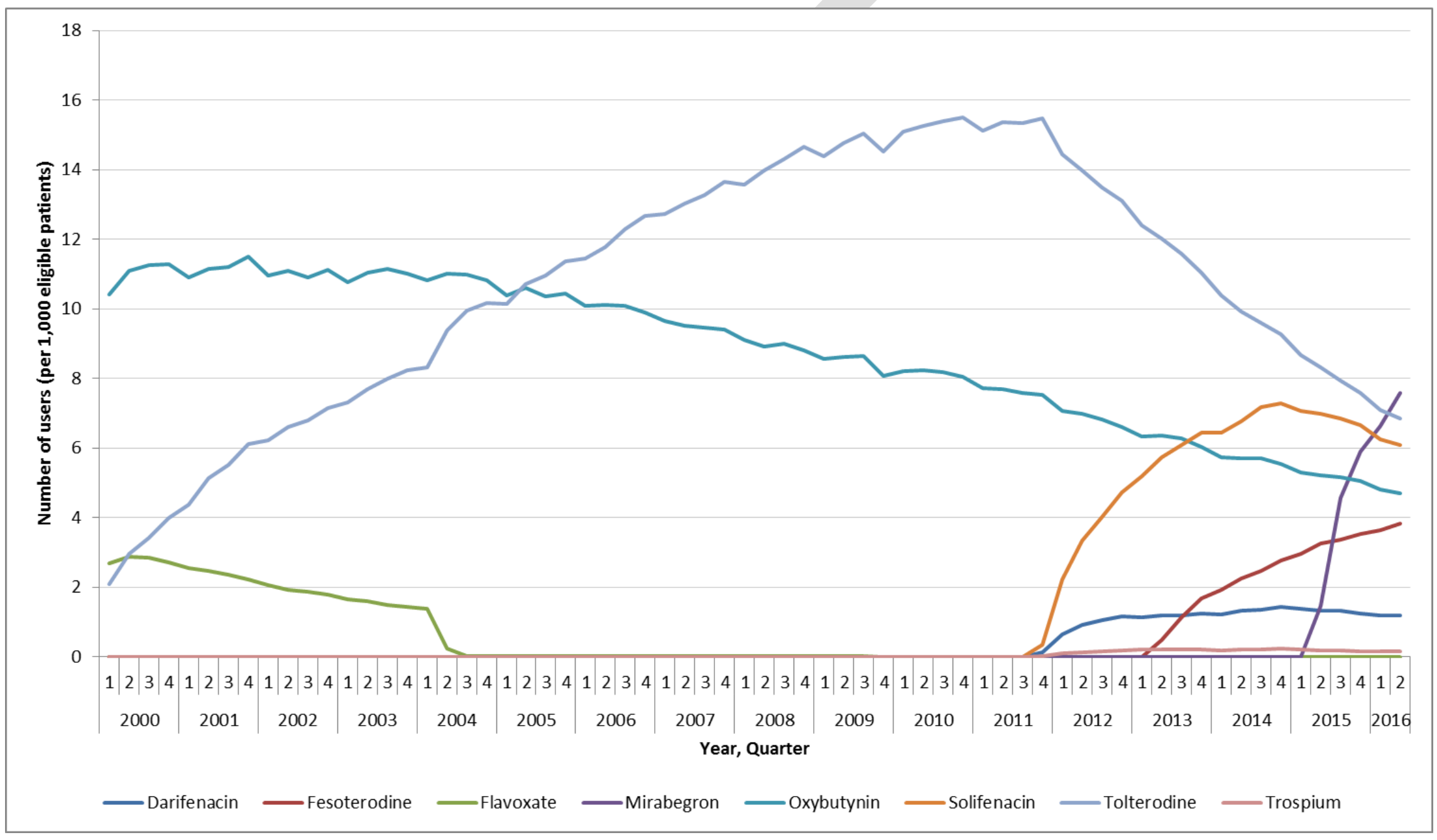


Tadrous et al

OAB treatment patterns

Fig. 2. Proportion of publically funded overactive bladder treatment users by drug in Ontario from 2000-2016.
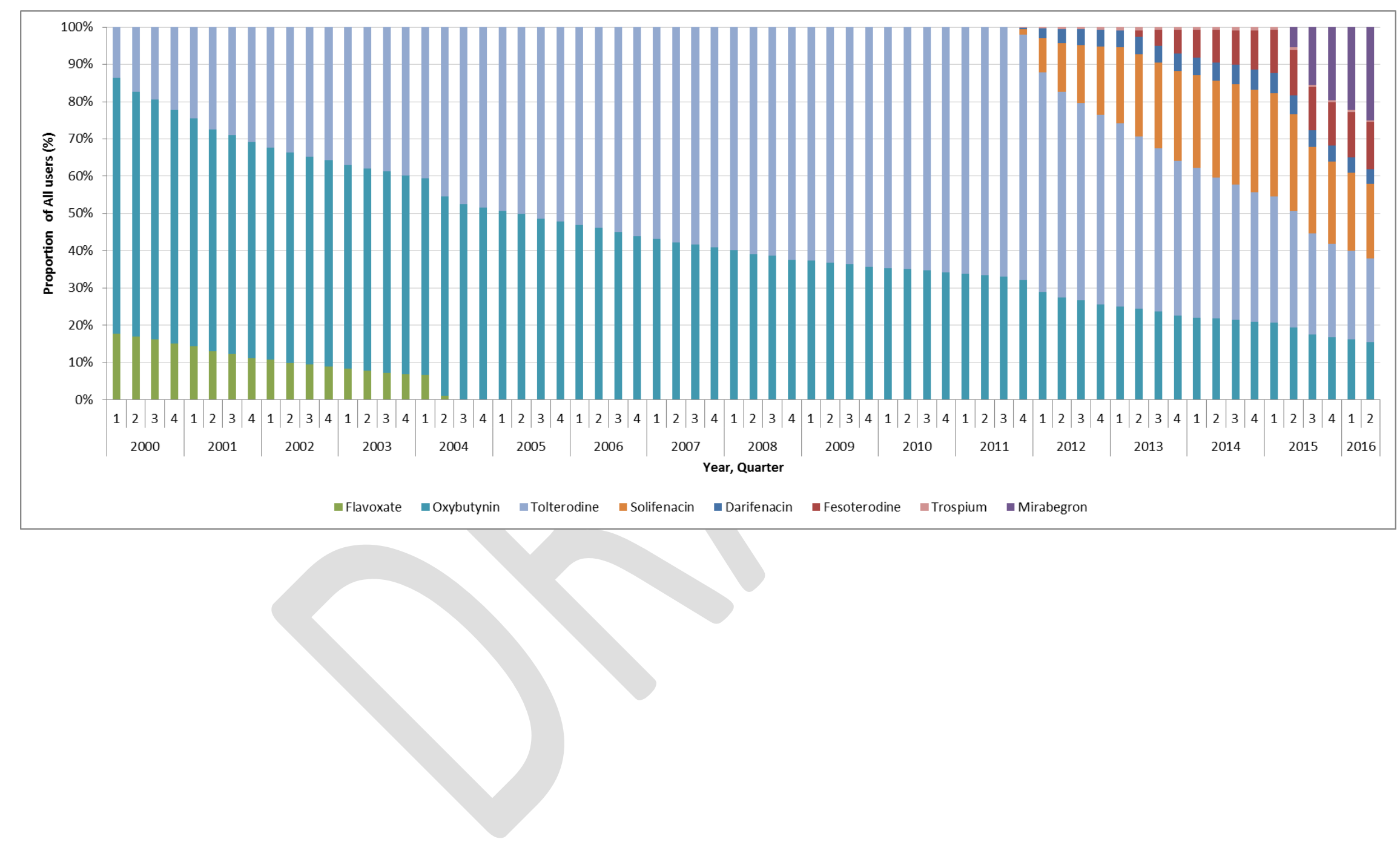